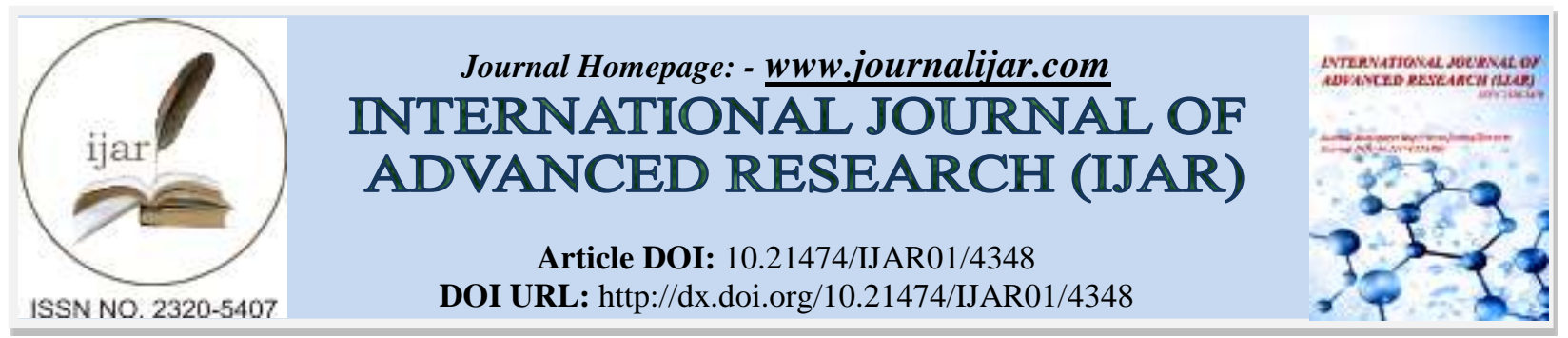

RESEARCH ARTICLE

\title{
OPHTALMOMYIASE EXTERNE A OESTRUS OVIS A DAKHLA (MAROC): A PROPOS DE 5 CAS OPHTHALMOMYIASIS EXTERNAL TO OESTRUS OVIS IN DAKHLA (MOROCCO): ABOUT 5 CASES.
}

\author{
Y.Bennouk ${ }^{1}$, L.Ait haj ${ }^{2}$, A. chikabab ${ }^{3}$ and M. Kriet ${ }^{2}$. \\ 1. Serviced ophtalmologie de l'hôpital militaire Dakhla. \\ 2. Service d ophtalmologie de l’hôpital militaire Marrakech. \\ 3. Laboratoire de 1'hôpital militaire Dakhla.
}

\section{Manuscript Info}

[........................

Manuscript History

Received: 12 March 2017

Final Accepted: 16 April 2017

Published: May 2017

Key words:-

External human ophtalmomyiasis

Oestrus ovis, Inflammation of the ocular

surface.

\section{Abstract}

We report 5 cases of ophthalmomyiasis external to oestrus ovis in soldiers affected in different regions of the periphery of Dakhla. All were hospitalized in March 2017 and exhibited the same symptomatology. The originality of this article in the fact that only military personnel have been affected contrary to what is reported in the literature where this pathology is frequent in the breeding areas.

Copy Right, IJAR, 2016,. All rights reserved.

\section{Introduction:-}

L'ophtalmolmyiase externe est une affection rare de la surface oculaire, due aux larves des mouches. Parmi les plus fréquents, l'Oestrus ovis parasitent certains animaux (1). Il touche l'homme accidentellement avec comme pathologies oculaires ; conjonctivite, kérato-conjonctivite, cellulite pré-septale (2). Généralement de bon pronostic si on traite convenablement sinon risque de transformation en ophtalmomyiase interne, rare mais plus grave et de mauvais pronostic (3).

\section{Materiels et methodes:-}

Nous rapportons une série de 5 cas tous des militaires exerçant en périphérie de Dakhla, âgés de 26,27, 30, 32 et 34 ans, admis en urgence au service d'ophtalmologie de l’hôpital militaire de Dakhla le mois de Mars 2017.

Tous les patients rapportent la même symptomatologie : atteinte unilatérale avec œil rouge, sensation de corps étranger, prurit intense et larmoiement (figure1), suite à une contamination par dépôt d'œufs par la mouche adulte après un petit choc sur l'œil. L'inspection objective un œdème des paupière et une hyperhémie conjonctivale, l'examen à la lampe à fente retrouve des petites larves translucides avec des crochets noirâtres, mobiles et photophobes, au niveau de la paupière supérieure, de la cornée et du cul de sac conjonctival inférieur, la cornée est transparente avec test à la fluorescéine négatif, la chambre antérieure est optiquement vide et l'examen du segment postérieur après dilatation est sans signes particuliers éliminant ainsi une ophtalmomyiase interne.

Le traitement de première ligne a reposé sur l'extraction mécanique des larves et lavage oculaire au sérum salé : après instillation d'oxybuprocaine nous avons pu extraire 7 larves pour deux patients, 6 pour le troisième et 4 pour le quatrième, en associant un lavage oculaire et des voies lacrymales et un traitement topique à base d'antibiotique, corticoïde et agents mouillants. 
L'examen parasitologique a permis d'identifier une larve de mouche de stade 1 et qui correspond à l'oestrus ovis : (figure2)

Elle mesure 1,2 $\mathrm{mm}$, blanche et translucide avec deux crochets noirs et scléreux, recourbés en forme de corne dans la région buccale, ils sont articulés sur un squelette chitineux caractéristique. Le corps possède sur sa partie ventrale deux rangs de spinules. à la région anale se trouvent deux tubercules.

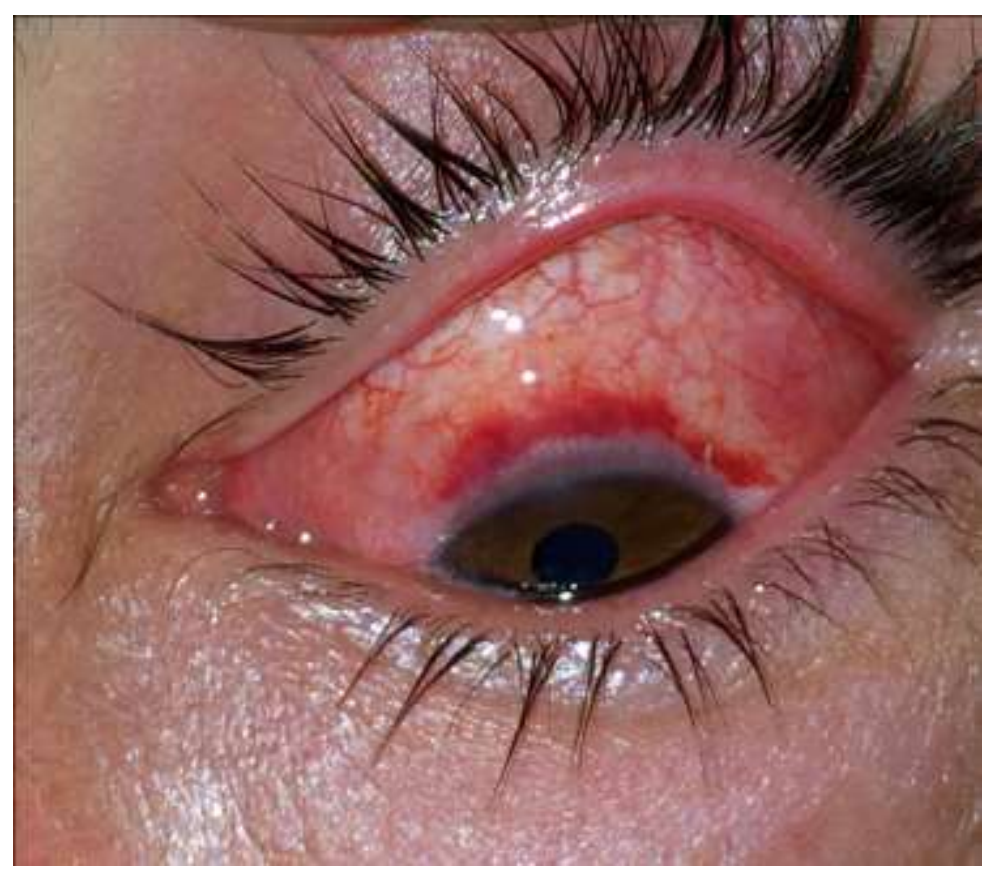

Figure 1:- patient presentant une ophtalmomyiase conjonctivale.



Figure 2:- larve d’oestrus ovis fixé sur une lame vu en microscopie optique. 


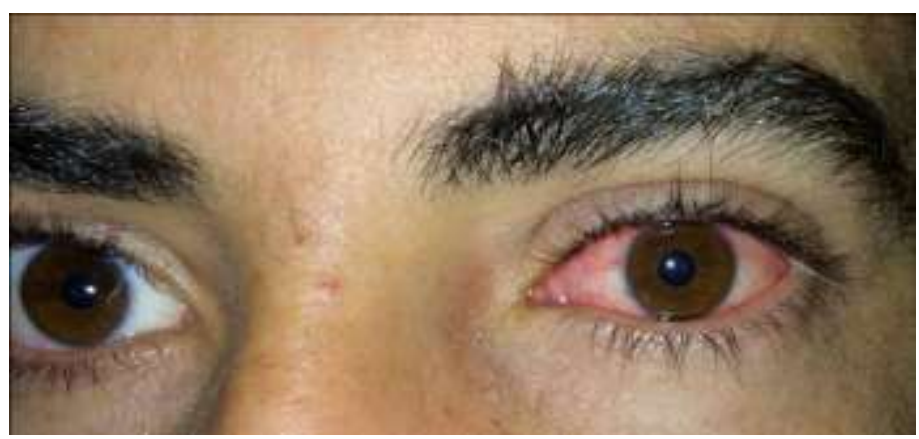

Figure 3:- patient à j1 du traitement.

\section{Resultats:-}

Tous les patients ont ressenti une nette amélioration $24 \mathrm{~h}$ après traitement (figure3). Les patients ont été garder en hospitalisation pendant $3 \mathrm{j}$ afin de s'assurer qu'on a extraire toutes les larves.

\section{Discussion:-}

L'ophtalmomyiase externe humaine est à peu près cosmopolite, mais semble plus fréquente dans les pays méditerranéens et le Proche-Orient $(3,4)$. On la rencontre essentiellement chez les populations vivant en contact direct avec les ongulés, ovins et caprins (5). Les bergers connaissent cette pathologie dont le nom commun varie selon les pays : «thimni » en Afrique du Nord, «sangalet» en Ethiopie, «sarticciolli » en Corse. La plupart du temps ils ne consultent pas et savent extraire eux-mêmes les larves, geste suivi d'une guérison sans séquelles, ce qui explique le nombre rare des cas rapporter dans la littérature (2).

L'ophtalmomyiase interne ne constitue que 5\% de l'ensemble des ophtalmomyise (3). Elle se caractérise par la présence du parasite en intra-oculaire avec souvent des complications sérieuses par destruction des tissus intraoculaires, mais peu aussi être dans les paupières et l'orbite (3).

L'oestrus ovis est une mouche dont le développement larvaire se fait obligatoirement sur des hôtes intermédiaires (moutons et chèvres) (6), la mouche adulte d'oestrus ovis est ovovivipare ; elle dépose en vol ses larves de premier stade autour des nasaux des animaux. Les larves rampent vers les cavités nasales et les sinus. Elles peuvent y rester jusqu'à 10 mois en se nourrissant d'exsudats. Elles passent par trois stades de développement. La larve stade I reste dans les cavités. La larve stade II migre dans les sinus frontaux où elle se transforme en larve de stade III. Dès qu'elles arrivent à maturité les larves de troisième stade sortent par les narines et tombent sur le sol où elles se transforment en pupes. Quand la température et l'humidité sont optimales, les adultes apparaissent au bout d'une dizaine de jours. La mouche adulte peut vivre de quelques jours à 2 mois. On la rencontre du printemps à l'automne $(5,7)$.

La contamination de l'homme s'effectue accidentellement par dépôt des larves par la mouche femelle classiquement dans 1'œil, moins fréquemment dans les oreilles, le nez et la gorge (8).

Chez nos patients la contamination (mois de mars) et les signes décrits sont classiquement les mêmes dans la littérature. La différence réside que tous les patients sont militaire et vivent loin de toute zone d'élevage. Le nombre peu des larves, retrouvées chez nos patients, est expliqué par le lavage sur place au sérum salé effectué au niveau des infirmeries des casernes.

\section{Conclusion:-}

L'ophtalmomyiase externe est une affection bénigne de la surface oculaire dont le traitement primordial est d'extraire toutes les larves présentes dans la conjonctive, sans oublier de laver les voies lacrymales afin d'éviter la pénétration intra-oculaire responsable d'un tableau rare d'ophtalmomyiase interne de mauvais pronostic et qui fait appel à un traitement plus lourd.

Personne ni à l'abri d'où une éducation sanitaire de la population ou des militaires qui travaillent dans des casernes en milieu rural s'impose, en insistant sur les mesures d’hygiène. 


\section{Conflits et intérêts:-}

Les auteurs déclarent ne pas avoir de conflit d'intérêt en relation avec cet article.

\section{Bibliographie:-}

1. N.Albaroudi, M. Tijani, R.Daoudi. Ophtalmomyiase externe au Maroc : à propos d'un cas en milieu urbain. JFO $2016 ;,-$.

2. J.Suzzoni-Blatger, L.villeneuve, B.Morassin, J. Chevallier. J Fr .Ophtalmol., 2000 ; 23,10,1020-1022.

3. R.Khoumiri, L.Gaboune, A.Sayouti, A.Moutaouakil. Ophtalmomyiase interne : à propos de deux cas. J Fr Ophtalmol., 2008 ; 31, 3, 299-302.

4. Dorchies Ph. Physiopathologie comparée de la myiase à Oestrus ovis(Linné 1761) chez l’homme et chez les animaux. Bull Acad Nat Méd, 1997 ; $181: 673-84$.

5. Yilma JM, Dorchies Ph, Epidemiology of Oestrus ovis in south-west France. Vet. Parasitol, $1991 ; 40: 315-23$.

6. Rodhain F, Perez C. les diptères myiasigènes In : Précis d'entomologie médical et vétérinaire. Paris : Maloine ed ; 1985. P. 249-65.

7. Dorchies Ph, Larrouy G, Deconinck P, Chantal J. L'ophtalmomyiase externe humaine : revue bobliographique à propos de cas en République de Djibouti. Bull Soc Path Exot, 1995 ; 88 : 86-9.

8. Brisou P, Menard G. Ophtalmomyiase externe à Oestrus ovis sur une plage varoise. Med. Trop, $2000 ; 60: 64-$ 6. 